REVIEW

\title{
Tropical pyomyositis (myositis tropicans): current perspective
}

\author{
S Chauhan, S Jain, S Varma, S S Chauhan
}

Postgrad Med J 2004;80:267-270. doi: 10.1136/pgmj.2003.009274

Tropical pyomyositis, a disease often seen in tropical countries, is characterised by suppuration within skeletal muscles, manifesting as single or multiple abscesses. The most common organism implicated is Staphylococcus aureus. In $20 \%-50 \%$ of cases there is a history of trauma to the affected muscles. Commonly involved muscles are quadriceps, glutei, pectoralis major, serratus anterior, biceps, iliopsoas, gastrocnemius, abdominal and spinal muscles. Early diagnosis is often missed because of lack of specific signs, unfamiliarity with the disease, atypical manifestations, and a wide range of differential diagnosis. Diagnostic techniques like ultrasound and computed tomography/magnetic resonance imaging are very useful in diagnosis. The diagnosis is confirmed either by biopsy or aspiration of pus from the affected muscles. The initial antibiotic of choice is cloxacillin. Incision and drainage are important components of management. Treatment for Gram negative or anaerobic organisms should be instituted, whenever indicated. Physicians should become more familiar with this potentially life threatening but curable infective disease entity.

See end of article for authors' affiliations

Correspondence to: Dr Sandeep Chauhan, 126A Sector 6, Panch'kula 134109, Haryana, India; drsc69@hotmail.com

Submitted 23 April 2003 Accepted

4 December 2003
$\mathrm{T}$ raquair credited Virchow for the earliest mention of tropical pyomyositis, ${ }^{1}$ though Scriba in 1885 described this entity for the first time. ${ }^{2}$ Levin et al in 1971 reported the first case from a temperate region. ${ }^{3}$ Since then many cases have been reported from various geographical regions of the world..$^{4-9}$ This increase in incidence is attributed to heightened awareness of this disease, an increase in the number of immunocompromised patients, and improvement in diagnostic techniques.

Since this entity is characteristically found in tropical areas, ${ }^{6-9}$ various terms like tropical pyomyositis, myositis tropicans, tropical skeletal muscle abscess, and tropical myositis are used. With increasing recognition from temperate regions, it is also referred to as non-tropical myositis, infectious myositis, or spontaneous bacterial myositis. Although the classical presentation is with muscle abscess, the hallmark of the disease is not an abscess but finding myositis on a biopsy specimen of involved muscle. Therefore, some authors prefer the term myositis instead of pyomyositis. ${ }^{10}$ The term "tropical pyomyositis" should be restricted to primary muscle abscess arising within the skeletal muscle. It should not be used to describe (a) intermuscular abscesses, (b) abscesses extending into muscles from adjoining tissues such as bone or subcutaneous tissues, and (c) those secondary to previous septicaemia.

\section{PREVALENCE AND INCIDENCE}

Tropical pyomyositis is common throughout the tropics and has been widely reported from Asia, tropical Africa, Oceania, and the Caribbean islands. It accounts for $1 \%-4 \%$ of all hospital admissions in some tropical countries. ${ }^{311}$ In Uganda about $4 \%$ of all hospital admissions and in Ecuador $2.2 \%$ of all surgical admissions have been reported as due to tropical pyomyositis. ${ }^{12}{ }^{13}$ In India it has not been widely reported but there have been sporadic case reports. ${ }^{14} 15$ Tropical pyomyositis is also being increasingly reported from temperate regions. ${ }^{5}{ }^{16}$ Recent data indicate that up to $75 \%$ cases of tropical pyomyositis in temperate areas are immunocompromised $^{17}$ - HIV infected, or with diabetes, leukaemia, chronic renal failure, asplenia, scleroderma, rheumatoid arthritis, or Felty's syndrome-while some patients have been on cancer chemotherapy or immunosuppressive drugs after transplantation of solid organs. Tropical pyomyositis has also been described after bicycle accidents and vigorous exercise. ${ }^{18} 19$ The incidence of tropical pyomyositis in HIV infected patients with or without AIDS is around $31 \%{ }^{20-22}$ Although cases are seen throughout the year, maximum incidence is from July to September (the monsoon season). ${ }^{23}$

\section{AETIOLOGY}

Staphylococcus aureus is the organism most commonly cultured from the abscess. It is seen in up to $90 \%$ of cases in tropical areas and $75 \%$ of cases in temperate countries. ${ }^{5}$ Group A streptococcus accounts for another $1 \%-5 \%$ of cases and other organisms uncommonly implicated are streptococcus (groups B, C, G), pneumococcus, neisseria, haemophilus, aeromonas, serratia, yersinia, pseudomonas, klebsiella, and escherichia. ${ }^{54}$ Rarely salmonella, citrobacter, fusobacterium, anaerobes, and mycobacterium are seen..$^{25-29}$

In tropical regions, pus cultures are sterile in $15 \%-30 \%$ cases $^{10}$ and $90 \%-95 \%$ of patients also, have sterile blood cultures. ${ }^{24}{ }^{30}$ Blood cultures are positive in $20 \%-30 \%$ of cases in temperate regions. ${ }^{31}$ Better microbiological culture techniques in the temperate regions may account for these variations. 


\section{PATHOGENESIS}

Pyomyositis is an intriguing disease of unclear pathogenesis. Organisms reach skeletal muscles during transient bacteraemia. Skeletal muscle tissue is intrinsically resistant to bacterial infections under normal circumstances. ${ }^{32}$ It is thought that there is sequestration of iron by myoglobin, which is an essential nutritional requirement of proliferating bacteria. This results in slower growth of bacteria, allowing cellular and humoral defences to enter infected zones and thereby preventing establishment of infection. In a series of autopsied cases of staphylococcus septicaemia, abscesses in skeletal muscle were found in fewer than $1 \%$ of cases. ${ }^{33}$ Even on direct inoculation in the muscles of dogs, staphylococcus failed to produce an abscess. ${ }^{34}$ Normal muscle, if damaged, is susceptible to haematogenous invasion by bacteria with subsequent abscess formation. ${ }^{35}$ In $20 \%-50 \%$ of cases, a history of blunt trauma or vigorous exercise of the involved group of muscles is forthcoming. ${ }^{36}$ Other postulated mechanisms, though not proven, include nutritional deficiencies and viral and parasitic infections. It is postulated that abundant iron is available after trauma, resulting in profuse growth of bacteria.

It has also been suggested that an abnormality of the immune system may be the underlying cause in many cases. Some researchers have proved that the lymphocytes, particularly T-cells, in patients with tropical pyomyositis are not primed adequately against staphylococcus during the course of infection. ${ }^{37}$ Other predisposing factors implicated include a preceding viral infection (especially arbovirus) or nematode infection (Dracunculus medinensis) but their role in pathogenesis is uncertain. ${ }^{38}$ Moreover, as these abscesses are intermuscular rather than intramuscular, these should not be included under tropical pyomyositis.

Intravenous drug abuse is another important risk factor for tropical pyomyositis. ${ }^{39}{ }^{40}$ The factors responsible are impaired cellular and humoral immunity, defective bactericidal capacity of neutrophils, increased bacterial colonisation of skin, and direct injection of contaminated materials.

Tropical pyomyositis is also increasingly documented in persons infected with HIV. ${ }^{20} 4142$ Mechanisms include muscle damage caused by HIV infection per se, zidovudine therapy, infections caused by parasites and mycobacteria, and impaired host defences including dysfunction of helper Tcells and granulocytes. ${ }^{43}{ }^{44}$ In some studies, it has been demonstrated that the CD4 counts are less then 150/ $\mu$ l in such cases. ${ }^{45}{ }^{46}$ Also, those with HIV infection have an increased incidence of staphylococcus carrier state compared with those not infected with HIV. ${ }^{47}$

\section{PATHOLOGY}

In early stages of tropical pyomyositis, muscles show oedematous separation of fibres, followed by patchy myocytolysis progressing to complete disintegration. The fibres are surrounded by lymphocytes and plasma cells. Muscle fibres may heal without abscess formation or degenerate, progressing to suppuration with bacteria and polymorphonuclear leucocytes. ${ }^{48}$

\section{CLINICAL FEATURES}

The disease is seen in all age groups, although young males are the most susceptible group. Maximum incidence is seen at 10-40 years of age with a male to female ratio of 1.5:1. Muscles frequently involved are quadriceps, glutei, pectoralis major, serratus anterior, biceps, iliopsoas, gastrocnemius, abdominal and spinal muscles. ${ }^{49}$ Usually, a single group of muscle is affected, but in $12 \%-40 \%$ of cases multiple groups are involved either sequentially or simultaneously. ${ }^{6}{ }^{11}$ The clinical picture is divided into three stages. ${ }^{50}$

\section{(1) Invasive stage}

This first stage is characterised by a subacute onset of variable fever, painful firm swelling, and minimal systemic symptoms with or without erythema (as infection is deep seated). The area is tender with a wooden consistency. Aspiration, if attempted at this stage, yields no pus as the phlegmonous inflammatory process is diffuse. Firm swelling, absence of erythema, and mild pain may divert the attention of a physician away from an infectious aetiology. The invasive stage may resolve itself, mimicking fibromyalgia or may progress to next stage of suppuration.

\section{(2) Suppurative stage}

From the second week to third week, abscess forms in the muscle. High spiky fever, with more severe systemic symptoms marks the beginning of the suppurative stage. Most cases present at this time. The classical signs of abscess, fluctuation and erythema, may be lacking because the overlying muscle is tense. Needle aspiration at this stage yields pus. Regional lymph nodes are not involved.

\section{(3) Late stage}

If the abscess remains untreated, dissemination of infection occurs. Bacteraemia, followed by septicaemia, septic shock, acute renal failure, and metastatic abscesses are some of the complications described..$^{30} 5152$

\section{ATYPICAL PRESENTATIONS}

The patient may present acutely with fever and chills or local symptoms. In a rare patient, the picture may be that of toxic shock syndrome. ${ }^{53}$ At times the invasive stage may be prolonged and the patient may present with pyrexia of unknown origin. Rarely, it may present as an acute abdomen or spinal cord compression ${ }^{54}$ or compartment syndrome. ${ }^{55}$ When localised to neck muscles, it can be mistaken for cervicobrachial neuralgia. ${ }^{56}$

\section{DIFFERENTIAL DIAGNOSIS}

Tropical pyomyositis is a great masquerader. Its differential diagnosis is wide and includes pyrexia of unknown origin, muscle contusion, septic arthritis, osteomyelitis, cellulitis, muscle haematoma, deep vein thrombosis, muscle rupture or muscle strain, osteosarcoma of muscle, trichinosis, leptospirosis, and polymyositis. Occasionally, the stage of invasion by Cysticercus cellulosae is characterised by fever, muscle tenderness, and eosinophilia and needs to be differentiated from tropical pyomyositis. Rarely, focal or diffuse inflammatory myopathy due to trypanosomiasis or toxoplasmosis presents as severe myalgia mimicking pyomyositis. It must also be distinguished from spontaneous gangrenous myositis (caused by Streptococcus pyogenes) characterised by gangrenous necrosis. Therefore, a diagnosis of tropical pyomyositis should be considered in any patient presenting with muscle pain, fever, and/or leucocytosis.

\section{DIAGNOSIS}

Early diagnosis is critical for saving the tissue and also the life of patient but is often missed because of unfamiliarity with the disease, atypical presentations, a wide range of differential diagnoses, and lack of early specific signs. Aspiration of pus from the muscle or muscle biopsy with culture and tissue staining in cases of absent macroabscesses is the gold standard for diagnosis (pus may be sterile in 15\%-30\% of cases ${ }^{10}$ ). Muscle biopsy also helps to exclude osteosarcoma, polymyositis, trypanosomiasis, toxoplasmosis, cysticercosis, and trichinosis.

Non-invasive radiological methods are helpful in establishing the diagnosis. These techniques are also used for subsequent follow up of the patient. Ultrasound is the initial 
screening tool for reasons of economy and easy availability. Hypoechoic areas with an increase in muscle bulk are seen on ultrasound. ${ }^{57}$ Computed tomography/magnetic resonance imaging (MRI) are the best imaging techniques for early diagnosis. ${ }^{58}$ Computed tomography shows areas of low attenuation with loss of muscle planes and a surrounding rim of contrast enhancement as characteristic of pyomyositis. Computed tomography is also useful in differentiating tumours, haematoma, and thrombophlebitis from abscess. ${ }^{59}$ However, at times a computed tomogram alone may be unreliable in distinguishing muscle abscess from swollen muscles. ${ }^{60}$ MRI shows hyperintense rim on Tl weighted images with peripheral enhancement on gadolinium DTPA scan. ${ }^{57}{ }^{61}$ A case has been reported in which areas of signal attenuation were visible on MRI but a computed tomogram was normal. ${ }^{62}$ Also, MRI is the imaging modality of choice for pelvis. ${ }^{57}$ Therefore, MRI may be a better modality than computed tomography for early diagnosis. Gallium scintigraphy is an extremely sensitive tool, but its inability to provide anatomic details, lack of specificity, and high cost reduces its usefulness. It is best reserved for a group of patients in which clinical suspicion is high but computed tomography/MRI is inconclusive. It is also utilised for detecting unsuspected but possible metastatic abscess. ${ }^{63}{ }^{64}$

Laboratory investigations reveal anaemia, leucocytosis (shift to the left), raised erythrocyte sedimentation rate, and acute phase reactants. In an asymptomatic patient with muscle mass and no other features of infection (fever, chills, erythema) but who has leucocytosis and raised erythrocyte sedimentation rate, pyomyositis should be suspected. In cysticercosis and trichinosis, eosinophilia is present. Blood cultures have low positivity, varying from $5 \%-10 \%$ in the tropics to $20 \%-30 \%$ in temperate regions ${ }^{30}{ }^{31}$ but are essential in all suspected or proven cases. Serum levels of muscle enzymes-that is, aldolase, creatine phosphokinase, aminotransferase, and lactic dehygrogenase-are normal or slightly raised despite evidence of muscle destruction. ${ }^{65}{ }^{66}$ Raised creatine phosphokinase along with characteristic electromyography changes (short duration, low amplitude polyphasic potentials), favour the diagnosis of polymyositis. Deranged liver and renal functions, along with fever and severe diffuse myalgia, indicate leptospirosis that is confirmed by serological tests. Immunodeficient states must be ruled out and tests for HIV, diabetes, rheumatological disorders, and malignancies should be carried out, along with immunoglobulin levels.

\section{TREATMENT}

Once diagnosis is established, attention should turn to aggressive management. Surgical debridement and drainage, accompanied by parenteral antistaphylococcal $\beta$-lactamase resistant penicillin (cloxacillin $1-2 \mathrm{~g}$ every six hours), is the initial recommended treatment. Diffuse myositis without abscess may respond to an antimicrobial agent alone but abscess may develop eventually requiring drainage. In most clinical settings, treating $S$ aureus infection with more than one drug, to which the organism is known to be susceptible, attains no significant benefit. Penicillin is the drug of choice for infections caused by penicillin susceptible staphylococcus. Drug combinations of penicillin with $\beta$-lactamase inhibitor are also effective but are best reserved for treatment of mixed infections. In case of penicillin allergic subjects, first generation cephalosporin (cefazolin) may be preferred for reasons related to cost, potency, and breadth of spectrum. For methicillin resistant staphylococcus, vancomycin in a dose of $15 \mathrm{mg} / \mathrm{kg}$ to a maximum of $1 \mathrm{~g}$, given every 12 hours is a suitable alternative. Another glycopeptide, teicoplanin, in a dosage of $400 \mathrm{mg} /$ day in a single dose is equally effective. For vancomycin intermediate sensitive staphylococcus, linzolid or dalfopristine-quinapristine are effective alternatives. ${ }^{67}$
If group A streptococcus is isolated from the pus, treatment should be changed to crystalline penicillin, while awaiting culture and sensitivity reports. ${ }^{68}$ For Gram negative bacilli, addition of an aminoglycoside, that is, gentamicin in doses of $5-6 \mathrm{mg} / \mathrm{kg} /$ day intravenously, should be considered in addition to cephalosporins. The possibility of associated anaerobic infection is an indication for introducing metronidazole (20$30 \mathrm{mg} / \mathrm{kg} /$ day) intravenously or orally at intervals of eight hours.

For HIV infection and immunosuppressed patients, broad spectrum empirical antibiotics against Gram positive, Gram negative and anaerobic organisms should be administered. In addition to antistaphylococcal antibiotics, patients should also receive aminoglycosides and clindamycin.

Secondary spread of metastatic infection from involved muscles usually requires four to six weeks of parenteral high dose antimicrobial therapy. Otherwise, treatment should be continued till wound is clean, the leucocyte count becomes normal, and the patient is afebrile for 7-10 days. Continuation or recurrence of fever after surgical drainage while the patient is receiving appropriate antimicrobials suggests the presence of other foci, development of drug resistance or, less commonly, drug fever.

Although there are no recommendations to prevent pyomyositis, it is believed that nasal carriage should be eliminated in patients with a history of pyomyositis or bacteraemic staphylococcal infection. ${ }^{69}$ It should be treated with topical mupirocin nasal formulation. Alternatives include rifampicin (600 mg each day) or cloxacillin (500 mg four times a day) for 10 days.

\section{PROGNOSIS}

The natural history is progressive suppuration with either spontaneous drainage and gradual resolution or eventual bacteraemia, and secondary infection leading to fatal outcome. Despite advances in diagnosis and treatment, mortality varying from $0.5 \%$ to $2 \%$ still occurs. ${ }^{712}$

\section{Authors' affiliations}

S Chauhan, S Jain, S Varma, Department of Internal Medicine, PGIMER, Chandigarh, India

S S Chauhan, City Medical Centre, Panchkula, Haryana, India

\section{REFERENCES}

1 Traquair RN. Pyomyositis. J Trop Med Hyg 1947;50:81-9.

2 Scriba J. Beitrang zur, Aetiologie der myositis acuta. Deutsche Zeit Chir 1885;22:497-502.

3 Levin MJ, Gardner P, Waldvogel F. Tropical pyomyositis: an unusual infection due to Staphylococcus aureus. N Engl J Med 1971;24:196-8.

4 Gibson RK, Rosenthal SJ, Lukert BP. Pyomyositis: increasing recognition in temperate climates. Am J Med 1984;77:768-72.

5 Christin L, Sarosi GA. Pyomyositis in North America: case reports and review. Clin Infect Dis 1992;15:668-77.

6 Chacha PB. Muscle abscesses in children. Clin Orthop 1970;70:174-80.

7 Cheidozi LC. Pyomyositis: review of 205 cases in 112 patients. Am J Surg 1979:137:255-9

8 Foster WD. The bacteriology of tropical pyomyositis in Uganda. J Hyg 1965;63:517-24

9 Ladipo GO, Fakunle YF. Tropical pyomyositis in the Nigerian Savanna. Trop Geogr Med 1977;29:223-8.

10 Shepherd JJ. Tropical myositis, is it an entity and what is its causes? Lancet 1983;ii:1240-2.

11 Horn CV, Master S. Pyomyositis tropicans in Uganda. E Afr Med J 1968;45:463-71.

12 Smith PG, Pike MC, Taylor E, et al. The epidemiology of tropical pyomyositis in Mengo districts of Uganda. Trans R Soc Trop Med Hyg 1978;72:46-53.

13 Kerrigan KR, Nelson SJ. Tropical pyomyositis in eastern Ecuador. Trans R Soc Trop Med Hyg 1992;86:90-1.

14 Anand AC, Narayanan VA, Kalra AS, et al. Tropical pyomyositis with agammaglobulinemia. J Assoc Physicians India 1986:34:745-6.

15 Malhotra P, Singh S, Sud A, et al. Tropical pyomysitis-experience of a tertiary care hospital in North West India. J Assoc Physicians India 2000:48:1057-60

16 Bonafede $\mathbf{P}$, Butler J, Kimbrough R, et al. Temperate zone pyomyositis. West J Med 1992;156:419-23. 
17 Hossain A, Reis ED, Soundararajan K, et al. Nontropical pyomyositis: analysis of eight patients in an urban center. Am Surg 2000;66:1064-6.

18 Jayoussi R, Bialik V, Eyal A, et al. Pyomyositis caused by vigorous exercise in a boy. Acta Paediatr 1995;84:226-7.

19 Diamandakis V, Grose C. Bad consequences of bicycle accidents. Pyomyositis. Pediatr Infect Dis J 1994;13:422-5.

20 Rodgers WB, Yodlowski ML, Mintzer CM. Pyomyositis in patients who have human immunodeficiency virus: case report and review of literature. J Bone Joint Surg Am 1993;75:588-92.

21 Ansaloni L. Tropical pyomyositis. World J Surg 1996;20:613-7.

22 Ansaloni L, Acaye GL, Re MC. High HIV sero-prevalence among patients with pyomyositis in northern Uganda. Trop Med Int Health 1996;1:210-2.

23 Singh SB, Singh VP, Gupta S. Tropical myositis a clinical, immunological and pathological study. J Assoc Physicians India 1989;37:561-3.

24 Sarubbi FA, Gafford GD, Bishop DR. Gram negative bacterial pyomyositis: unique case and review. Rev Infect Dis 1989;11:789-92.

25 Mootsikapun P, Mahakkanukrauh A, Suwannaroj S, et al. Tuberculous pyomyositis. J Med Assoc Thai 2003;86:477-81.

26 Minami K, Sakiyama M, Suzuki H, et al. Pyomyositis of the vastus medialis muscle associated with Salmonella enteritidis in a child. Pediatr Radiol 2003:33:492-4.

27 Brook I. Pyomyositis in children, caused by anaerobic bacteria. J Pediatr Surg 1996;31:394-6.

28 Wolf RF, Konings JG, Prins TR, et al. Fusobacterium pyomyositis of the shoulder after tonsillitis. Report of a case of Lemierre's syndrome. Acta Orthop Scand 1991;62:595-6.

29 Fincher RM, Jackson MW, Fischer AQ. Citrobacter freundii: a newly reported cause of pyomyositis. Am J Med Sci 1990;299:331-3.

30 Gambhir IS, Singh DS, Gupta SS, et al. Tropical pyomyositis in India, a clinico-histopathological study. J Trop Med Hyg 1992;95:42-6.

31 Brown JD, Wheeler B. Pyomyositis: report of 18 cases in Hawaii. Arch Intern Med 1984; 144:1749-51.

32 Grandsen WR, Ekysyn SJ, Philips I. Staphylococcus aureus bacteraemia, 400 episodes. BMJ 1984;288:300-5.

33 Smith MI, Vickers $A B$. Natural history of treated and untreated patients with septicaemia. Lancet 1960;i:1318-22.

34 Halsted WS. Surgical papers. Baltimore, MD: John Hopkins University Press, 1924:103.

35 Miyakae H. Beiterage zur kenntnis sogenannten myositis infectiosa. Milt Grenzzgeb Med Chir 1904;13:155-8.

36 Hall RL, Callaghan JJ, Moloney E, et al. Pyomyositis in a temperate climate, presentation, diagnosis and treatment. J Bone Joint Surg Am 1990;72:1240-4

37 Idoko JA, Oyeyinka GO, Giassuddin AS, et al. Neutrophil cell function and migration inhibition study in Nigerian patients with tropical pyomyositis. $J$ Infect 1987; 15:33-7.

38 Warrel DA. Tropical pyomyositis. In: Benz EJ, Firth JD, Cox TM, et al. Oxford textbook of medicine. 4th Ed. Oxford: Oxford University Press, 2003;3:1251-2.

39 Ebright JR, Pieper B. Skin and soft tissue infections in injection drug users. Infect Dis Clin North Am 2002;16:697-712.

40 Crossley M. Temperate pyomyositis in an injecting drug misuser. A difficult diagnosis in a difficult patient. Emerg Med J 2003;20:299-300.

41 Biviji AA, Paiement GD, Steinbach LS. Musculoskeletal manifestation of human immunodeficiency virus infection. J Am Acad Orthop Surg 2002; 10:312-20.

42 Ansaloni L, Acaye GL. Absence of neutropenia in African patients with AIDS and associated pyomyositis. East Afr Med J 1994;71:736-8.

43 Nielsen $H$, Kharzami A, Faber V. Blood monocyte and neutrophil functions in AIDS. Scand J Immunol 1986;24:291-6.
44 Philip MH Lane C, Fauci AS et al Impairment of neutrophil bactericidal capacity in patients with AIDS. J Infect Dis 1988;158:627-30.

45 Casado E, Olive A, Holgado S, et al. Musculoskeletal manifestations in patients positive for human immunodeficiency virus: correlation with CD4 count. J Rheumatol 2001 28:802-4.

46 Vassilopoulos D, Chalasani P, Jurado RL, et al. Musculoskeletal infections in patients with human immunodeficiency virus infection. Medicine Baltimore 1997;76:284-94

47 Ganesh R, Castle D, Mc Gibban D, et al. Staphylococcus infection and HIV carriage. Lancet 1989;ii:558.

48 Taylor JF, Templeton AC, Henderson B. Pyomyositis: a clinic-pathological study based on 19 autopsy cases, Mulango Hospital 1964-1968. East Af Med J 1970;47:493-501.

49 Ashken MH, Cotton RE. Tropical skeletal muscle abscesses (pyomyositis tropicans). Br J Surg 1963;50:846-52.

50 Chukawama CL. Pyomyositis. Am J Surg 1979;137:255-9.

51 Andy JJ, Ekpo EB. Cardiovascular complications of tropical pyomyositis. Trop Geogr Med 1987;39:260-4

52 Adeloye A. Intracranial suppuration complicating tropical pyomyositis: report of two cases. Trans R Soc Trop Med Hyg 1982;76:463-4.

53 Immerman RP, Greenman RL. Toxic shock syndrome associated with pyomysitis caused by a strain of Staph aureus that does not produce toxic shock syndrome toxin. J Infect Dis 1987;156:505-7.

54 Brik R, Braun J, Bialik V, et al. Non-tropical pyomyositis in children-with report of severe neurological complications. Acta Paediatr Scand 1989;78:331-4

55 Onder C, Kalaycioglu A. Anterior tibial compartment syndrome due to the pyomyositis in a patient with rheumatoid arthritis. A case report. Joint Bone Spine 2003;70:77-9.

56 Flory $\mathbf{P}$, Brocq O, Euller-Ziegler L, et al. Pyomyositis: cervical localization. $J$ Rheumatol 1993;20:1411-3.

57 Trusen A, Beissert M, Schultz G et al. Ultrasound and MRI features of pyomyositis in children. Eur Radiol 2003:13:1050-5.

58 Gordon BA, Martinez S, Collins AJ. Pyomyositis: characteristics at CT and MR imaging. Radiology 1995;197:279-86.

59 Shumacher TM, Genant KH. Dorobion M, et al. Computed tomography. J Bone Joint Surg 1978;60:600-7.

60 Falasca GF, Reginato AJ. The spectrum of myositis and rhabdomyolysis associated with bacterial infection. J Rheumatol 1994;21:1932-7.

61 Soler R, Rodriguez E, Aguilera C, et al. Magnetic resonance imaging of pyomyositis in 43 cases. Eur J Radiol 2000;35:59-64.

62 Yuhi WT, Schreiber AE, Montgomery WJ, et al. Magnetic resonance imaging of pyomyositis. Skeletal Radiol 1988;17:190-3.

63 Pretorious ES, Hruban RH, Fishman EK. Tropical pyomyositis: imaging findings and review of literature. Skeletal Radiol 1996;25:576-9.

64 Schiff RG, Silver L. Tropical pyomyositis: demonstration of extent and distribution of disease by gallium scintigraphy. Clin Nud Med 1990; 15:542-4.

65 Gupta B, Khanna SK, Sharma BK. Pyomyositis. J Assoc Physicians India 1980;28:91-4.

66 Meena AK, Rajashekhar S, Reddy JJ, et al. Pyomyositis-clinical and MRI characteristics report of three cases. Neurol India 1999;47:324-6.

67 Robert L, Reresiewiscz, Parosenet J. Staphylococcal infections. In: Fauci AS, Braunwald E, Martin JB, et al. Harrison's principles of internal medicine. 15th Ed. New York: McGraw-Hill, 2001;1:889-900.

68 Weasels MR. Streptococcal infections. In: Fauci AS, Braunwald E, Martin JB, et al. Harrison's principles of internal medicine. 15th Ed. New York: McGraw Hill, $2001 ; 1: 901-9$.

69 Hudson IR. The efficacy of intranasal mupirocin in the prevention of staphylococcal infections: a review of recent experience. J Hosp Infect 1994:27:81-98. 